\title{
Cosmic rays, stellar evolution, and supernova physics
}

\author{
Peter L. Biermann ${ }^{\mathrm{a}, \mathrm{b}}$ \\ a Max-Planck Institute for Radioastronomy, Bonn, Germany \\ ${ }^{\mathrm{b}}$ Department for Physics and Astronomy, University of Bonn, Germany
}

\begin{abstract}
Most cosmic rays are thought to be accelerated by the shocks of supernova explosions of very massive stars. Here we review one quantitative proposal, which predicted the spectral slopes, bend and cutoff about the cosmic ray spectrum across the spectral bend or "knee" near $310^{15} \mathrm{eV}$ in 1993. Many of the specific predictions have now been verified, and so it may be appropiate to investigate the consequences of that proposal. The successful fit to the cosmic ray data across the knee suggests: 1 ) very massive stars, above about 20 to 25 solar masses all converge to a common final state; 2 ) the supernova explosion of these very massive stars is caused by a combination of rotation, magnetic fields and the gravitational potential, just as proposed by G. Bisnovatyi-Kogan in 1970, based on an earlier suggestion by N.S. Kardashev in $1964 ; 3)$ their stellar winds as well as the explosion provide the main injection of magnetic fields into the interstellar medium; 4) the explosive energy of these supernovae is about $\left.10^{52} \mathrm{erg} ; 5\right)$ the explosion is extremely anisotropic, which may unify several classes of observed supernova types; 6 ) gamma ray bursts may be the ultimate version of such an explosion in the case the collapse leads to a black hole; 7) it is plausible that the luminosity of the supernova integrated over all aspect angles is also the same for all these massive star explosions, leading to 8) a possibly very bright standard candle, if we could just find a correction for the extreme asphericity. This may solve the problem of what the mechanism of supernova explosions is for very massive stars, where most cosmic rays and magnetic fields come from, and may also point the way to a unifying scheme for supernova explosions and gamma ray bursts.
\end{abstract}

Key words: , stars: late stages of evolution, cosmic rays, supernovae: nucleosynthesis, magnetohydrodynamics and plasmas, electromagnetic fields, bursts

PACS: 97.60, 98.70, 26.30.+k, 95.30.Qd, 41.20, 98.62.N

Email address: plbiermann@mpifr-bonn.mpg.de (Peter L. Biermann).

Preprint submitted to New Astronomy Reviews 29 October 2018 


\section{Stellar evolution and Supernova explosions}

Massive stars form, evolve and finally explode as supernovae. The lower mass limit for single stars that explode is estimated to be near 8 solar masses. Stars in the mass range 8 to about 15 solar masses evolve without a strong stellar wind, and so explode directly into their environment, in the now classical picture of a self-similar explosion (e.g. D.P. Cox 1972). More massive stars, between about 15 and about 20 - 25 solar masses explode as red supergiant stars (RSG) with a wind. These winds are moderately powerful, and the surface of the star slowly exposes the deeper layers of Helium, so that the wind has a strong admixture of Helium at the time of explosion. From about 20 25 solar masses the stars evolve to a blue supergiant stage, the Wolf Rayet stage, with very powerful winds. These winds eat back into the star exposing the deep layers, and so show a mixture of Helium, Carbon and Oxygen. These winds are very strong, and produce a massive wind-shell, a shell of snow-plowed interstellar material, and former wind material, which in turn is of course former outer stellar material. The driving of these winds already had been a problem; it is generally accepted that radiation drives the wind, and that wave excitation transfers the momentum; the coupling constant is the wave speed. The temperature of the wind and so its speed of sound is strongly limited by cooling to 10,000 to 20,000 degrees K. Following earlier work by J. Cassinelli and St. Owocki we showed some time ago, that magnetic fields could help this problem by showing that the coupling between radiation and wind goes through the dominant wave speed, which could be the Alfvén speed; magnetic waves are not influenced by cooling. The optimum momentum transfer appears to occur when the wind is slightly super-Alfvénic, with an Alfvénic Mach number of a few, say, 3 (work with H. Seemann, 1997).

Wolf Rayet stars may hold the clue to our understanding of high energy cosmic ray particles, the origin of magnetic fields, the physics of supernova explosions, the origin of gamma ray bursts, and may even give us a bright standard candle for cosmology.

A much more extended recent version of some of these arguments is in two reviews by P.L. Biermann et al. (2003a, astro-ph/0302168, 2003b, astro-ph/0302201), two reviews based on talks at the Palermo meeting Sep 2002; these reviews also contain many important references, which are omitted here for lack of space. 


\section{The cosmic ray spectrum}

Cosmic ray particles, discovered in balloon flights just after 1910 by V.F. Hess and W. Kohlhörster, are now known to extend in particle energy to a few times $10^{20} \mathrm{eV}$. Around $310^{18} \mathrm{eV}$ there is a kink in the spectrum and also a change in the chemical compositon, and this is generally believed to be the transition between Galactic cosmic rays and extragalactic cosmic rays: However, a final proof for such an interpretation is still waiting to be discovered. Here we concentrate on the cosmic ray particles below $310^{18} \mathrm{eV}$, and ask what their sources may be.

So let us first summarize their properties:

The spectrum of cosmic rays is approximately $E^{-2.7}$ until the knee, which is a bend downwards at around $310^{15} \mathrm{eV}$. The spectrum beyond the knee is approximately $E^{-3.1}$. There is a slight downward dip from $310^{17} \mathrm{eV}$, and a transition near $310^{18} \mathrm{eV}$. At $310^{18} \mathrm{eV}$ there is switch from a quite steep local slope to a much flatter slope, as well as an apparent change in chemical composition from medium to heavy nuclei to light nuclei (Hydrogen and Helium). A general review including (almost) all data up to 1997 is in B. Wiebel-Sooth \& P.L. Biermann (1999).

The key features for Galactic cosmic rays are the high particle energies, and the spectral bend at the knee; we also need to understand the second "knee" at $310^{17} \mathrm{eV}$, where the spectrum dips to merge into the extragalactic cosmic rays near $310^{18} \mathrm{eV}$. Berezinsky et al. (2003) have recently discussed a very similar explanation of this dip.

Supernova explosions into the interstellar medium do not give such a kink, and also are not usually accepted to give a maximum particle energy of $310^{18} \mathrm{eV}$ (P. O. Lagage \& C. J. Cesarsky 1983). The kink at around $310^{15} \mathrm{eV}$ has had a variety of proposals to explain it: The main proposal has been the notion that it reflects a change in propagation: This entails a steepening of the energy dependence of the propagation by about 0.3 to 0.4 at a specific energy/charge ratio $E / Z$. Such a specific energy/charge ratio $E / Z$ corresponds to a precise length scale in the interstellar medium, about 0.4 parsec. At that length scale the properties of the irregularities in the interstellar medium should change rather drastically. There is no such evidence. Also, if that were the case, the anisotropies near $10^{18} \mathrm{eV}$ should be large, and from the AGASA data at that energy we know that the anisotropy is small. All available evidence suggests that as regards cosmic ray transport the interstellar medium can be described by a Kolmogorov law from very small scales much below $0.4 \mathrm{pc}$ all the way

up to much larger scales, up to a few hundred pc. This is still a fraction of the scale of the hot gas, magnetic field and cosmic ray disk, which has a full 
width of about $4 \mathrm{kpc}$, as suggested by ROSAT data (Snowden et al. 1997). Therefore we conclude that the explanation of the kink has to be sought in the source.

In a series of papers (first qualitatively, H.J. Völk \& P.L. Biermann 1988, and then quantitatively in several papers starting with P.L. Biermann 1993) we have suggested that the key to understand the acceleration of higher energy particles was the recognition that many massive stars explode into their own wind. In a magnetic wind, such as the Solar wind (L.F. Biermann 1951), the magnetic field has a basic topology already suggested by E.N. Parker (1958), with $B_{r} \sim 1 / r 2, B_{\theta}=0$, and $B_{\phi} \sim \sin \theta / r$, an Archimedian spiral. Therefore the dominant magnetic field runs as $1 / r$ with radial distance $r$, and so the Larmor radius of any particle with some given energy/charge ratio $E / Z$ scales linearly with $r$. Any shock racing through the wind introduces also a scale from the snow-plow effect, of $r / 4$. Near the pole the acceleration is in a parallel magnetic field configuration (i.e. shock normal parallel to the locally prevailing magnetic field), which is the situation discussed by P.O. Lagage \& C.J. Cesarsky (1983): near the equator the acceleration is in the near perpendicular configuration as discussed by R. Jokipii (1987); the perpendicular configuration introduces also drift acceleration, while both configurations also need to include adiabatic lossses, and the density history of injection. The key is the introduction of the notion of the "smallest dominant scale" to describe the large scale turbulence in the unstable shock region, both in real space as in velocity space (P.L. Biermann 1993); the character of the turbulence in the shock region is the critical argument here, as recognized by many. The final result is the predicted spectrum of $E^{-8 / 3-0.02 \pm 0.02}$ below the knee, $E^{-3.07-0.07 \pm 0.07}$ above the knee, for wind-SNe; from matching the two acceleration regimes the knee energy, as well as the ankle energy, are $E_{\text {knee }}=Z e B(r) r\left(3 / 4 U_{s h} / c\right) 2$, and $E_{\text {ankle }}=Z e B(r) r$. The gradual cutoff with $Z$ gives the second knee, and then the steepening between $310^{17} \mathrm{eV}$ and $310^{18} \mathrm{eV}$. Both quantities, $E_{\text {knee }}$ and $E_{\text {ankle }}$, are constant with $r$ in a magnetic wind, considering most of $4 \pi$. Putting in numbers suggested in 1993 (see the review at the Calgary 23rd ICRC meeting, P.L. Biermann 1994) that the knee and ankle energies are $0.6 Z 10^{15} \mathrm{eV}$, and $Z 10^{17} \mathrm{eV}$, with a fair uncertainty due to a specific choice of numbers for stellar properties, that are hard to know.

Recently, with the advent of the KASKADE data we have been able to test the predictions, and determined in a direct fit the two spectra to $E^{-2.67}$, and $E^{-3.14}$, and the two energies to $E_{\text {knee }}=1.7 Z 10^{15} \mathrm{eV}$, and $E_{\text {ankle }}=2.2 Z 10^{17}$ $\mathrm{eV}$. A full scale Monte-Carlo CORSIKA fit to all vertical and slanted shower data available from KASKADE gives very similar numbers, but then with error bars: The two spectra are $E^{-2.65 \pm 0.03}$ and $E^{-3.25 \pm 0.04}$, and the knee energy comes out to $2.1 \pm 0.14 Z 10^{15} \mathrm{eV}$, while the ankle energy cannot be easily determined in this way, using a Monte-Carlo approach; all details are described in P.L. Biermann et al. (2003b). Therefore the predictions were confirmed 
quantitatively.

This implies that all sources must be very similar, because otherwise a source based model could not describe an overlap of many injection events. Since the asymptotic Parker magnetic field topology in our context implies that the rotation of the star is highly differential so as to allow a highly tangential field already at the surface, this finding from the fit also has consequences for the rotation of the star, since angular momentum loss is minimized.

\section{Origin of magnetic fields}

These stars and their winds are magnetic, and so inject magnetic fields into the interstellar medium. We noted above that the Alfvénic Machnumber is most likely of order 3. This entails that the termination shock of the wind injects magnetic fields into the environment that have already near $10 \%$ of equipartition values. Considering the origin of the magnetic field in the interstellar medium, and its topology, this may be all that is necessary in terms of strength - however, this does not produce any order in the field, and that requirement leads to very different considerations. One might ask what magnetic fields massive stars produce in analogy to the Sun: The convective interior will produce a magnetic field from the seed field (derived from the fact that surfaces of constant pressure and constant density do not generally coincide in a rotating system, and so drive an electric current, L.F. Biermann 1950, L.F. Biermann \& A. Schlüter 1951) and the dynamo mechanism (M. Steenbeck \& F. Krause, and E.N. Parker). This magnetic field is quite strong, and can readily be transported to the surface by meridional circulations, also known as Sweet-Eddington circulations. Therefore we conclude that magnetic field may be relevant in the evolution of massive stars, as well as to explain the interstellar magnetic fields. As galactic winds and radio galaxies transport these galactic fields into intergalactic space, such as clusters of galaxies, and sheets and filaments of the cosmic web of the galaxy distribution, it might just be that Wolf Rayet stars hold one of two keys to explain all magnetic fields in the universe - the other key is the explanation of the symmetry and order.

\section{Implications for the supernova mechanism}

If the fit says that all stars give the same knee and ankle energy, then this immediately suggests that explosion energy, magnetic field, and rotation are deeply connected, and are in fact always nearly the same number for each exploding star, above the zero age main sequence star mass of about $20-25$ solar masses. 
This strongly supports the concept proposed by G. Bisnovatyi-Kogan in 1970, that the explosion of these massive stars is caused by a combination of rotation, magnetic fields and potential energy, based on an an earlier suggestion by N.S. Kardashev in 1964. The idea is as follows, and reminds one immediately of many much more recent arguments about gamma ray bursts (especially the work by C. Wheeler and S. Woosley):

The core of the star rotates, and so when it collapses due to a lack of supporting pressure, it contracts into a small flat disk in rotational balance. This is at a few $10^{7} \mathrm{~cm}$. Since at that size the magnetic field would exceed the Landau level, this transfer of energy implies many rotation periods of energy injection during the approach to this size. The magnetic field is all sheared and wound up, and so transports angular momentum and the potential energy of the small disk to the outside, exploding the star. The energy of the rotating small disk constitutes the visible energy of the supernova, and is readily estimated to be $10^{51}$ to $10^{52} \mathrm{erg}$. When the small disk then finally collapses into a neutron star or into a black hole, then that last energy is radiated away as neutrinos, as observed.

This mechanism obviously is extremely anisotropic, and it can safely be predicted that supernovae following this concept appear very different if viewed from different angles. It is quite plausible that various supernova types might be unified in such a scheme, very much in analogy to the unified scheme long used in active galactic nuclei, where aspect angle is a major parameter. It can be expected that along the symmetry axis explosion speeds might be very much faster than along the equatorial belt, and so may explain various observations of very fast knots. It is not clear at this point just what the precise connection to gamma ray bursts could be; if this is also the general mechanism for gamma ray bursts, in addition to the main mechanism for massive star explosions or supernovae, then gamma ray bursts could be very much more frequent than commonly argued.

Now considering the transfer of energy and angular momentum from the small collapsed disk by magnetic fields, it is clear that this is fully symmetric along the rotation axis between "up" and "down", giving a saddle point instability. This entails, that the slightest asymmetry between the two sides will give rise to a powerful sling shot effect exactly along the rotational and symmetry axis, leading to a high speed pulsar, which flies just along the direction defined by its own rotational axis. This can be tested observationally, and would be a second powerful confirmation of the mechanism of G. Bisnovatyi-Kogan (see Bisnovatyi-Kogan \& Moiseenko 1992).

Also, there is another corollary: Using the abundances in the pre-supernova winds (taken from N. Langer) we can determine the connection between cosmic ray flux for some element like Carbon, and connect the appropiate supernova 
rate with the energy contained in cosmic rays for each single event. This leads to an estimate of order $10^{51}$ erg for each event in cosmic ray particles alone, and then using an inefficiency of 0.1 to an explosion energy of $10^{52} \mathrm{erg}$, already quite close to what has been called a Hypernova by B. Paczynski.

\section{Gamma ray bursts}

There is growing evidence that gamma ray bursts and supernovae might be connected (see, e.g., Uemara et al. 2003, Price et al. 2003, Hjorth et al. 2003, and many other comments and articles on GRB 030329). There are two simple possibilities: First, for extremely massive stars the supernova explosion might lead to a central black hole, and maybe just those stars also produce a gamma ray burst. In this case the frequency of gamma ray bursts might be much higher than one per million years in a normal galaxy. Second, it is also conceivable that a binary system is required, with the main star exploding triggering a gamma ray burst in the small neutron star companion leading to a collapse to a black hole. In this case the mass transfer towards the neutron star companion could dramatically increase just prior to the explosion, and so trigger a catastrophic collapse very close to the supernova explosion. In the first case the gamma ray burst would happen at exactly the same time as the supernova, with very high velocities along the line of sight, and in the second case the gamma ray burst would have to precede the actual supernova explosion, because otherwise the supernova explosion itself would dirty the gamma ray burst with baryonic matter beyond recognition. Maybe Nature realizes both options. The literature shows that there are many more possibilities.

A magnetic mechanism provides an interpretation of the extreme degree of gamma ray polarization found recently (Coburn \& Boggs 2003, GRB 021206).

If we could find a way to observe the traces of the last few gamma ray bursts in our Galaxy, then the time scale could be strongly restricted. In recent work with R. Engel, G. Medina-Tanco and G. Pugliese we have proposed that the AGASA excess of events at $10^{18} \mathrm{eV}$ in the Galactic Center region is such a trace.

\section{Consequences for cosmology}

To emphasize the conclusion from the cosmic ray data fit: The explosion energy has to be very nearly the same for each such star, as also the rotation, magnetic field and mass - all measured just prior to the explosion. The stellar evolution

of these stars leads to a common final state just prior to the explosion. 
This suggests that the light curve of the supernova may also be the same, if integrated over all aspect angles. Such an integration might be possible using infrared emission, echoing and polarization. This remains to be worked out and tested. On the other hand, if this correction for anisotropy could be done, then we would have a standard candle for use in cosmology which has the promise to be much brighter than the commonly used supernova type Ia.

\section{Conclusion}

Wolf Rayet stars are one key to understand the magnetic fields in the universe, the enrichment in heavy elements, and much of the observed cosmic rays; cosmic rays in turn suggest that the magneto-rotational mechanism by $\mathrm{G}$. Bisnovatyi-Kogan does explain the explosion of these stars, possibly providing a key to understand gamma ray bursts. The cosmic ray data imply that these stars converge to a common final state in their evolution, just prior to the explosion. Finally, if we could work out the corrections for anisotropy, these supernovae could be very bright new standard candles in cosmology.

\section{Acknowledgements}

PLB would like to acknowledge working on these questions most recently with S. Casanova, R. Engel, N. Langer, H.S. Lee, G. Medina-Tanco, S. Moiseenko, A. Ødegård, G. Pugliese, E.-S. Seo, S. Ter-Antonyan, and A. Vasile. He would also like to acknowledge very lively and useful discussions on these topics with I. Axford, Z. Berezhiani, V. Berezinsky, G. Meynet, G. Sigl and T. Stanev. These results were first introduced in this breadth at lectures in Paris spring 2002 and then at the Palermo meeting September 2002 (see below, on astro-ph), and PLB would like to express his appreciation to N. Sanchez and her colleagues for their continuing hospitality. Work with PLB is mainly being supported through the AUGER theory and membership grant 05 CU1ERA/3 through DESY/BMBF (Germany); further support for the work with PLB comes from the DFG, DAAD, Humboldt Foundation and the Naumann-Foundation (all Germany), grant 2000/06695-0 from FAPESP (Brasil) through G. Medina-Tanco, KOSEF (Korea) through H. Kang and D. Ryu, ARC (Australia) through R.J. Protheroe, a NATO-grant with S. Moiseenko (Russia), and European INTAS/ Erasmus/ Sokrates/ Phare grants. Finally, PLB is very grateful to the organizers of the meeting at Seeon May 2003, specifically R. Diehl, for the invitation to present these results. The author also wishes to thank S. Moiseenko for a critical reading of the article. 


\section{References}

[1] Berezinsky, V., Gazizov, A., Grigorieva, S. (2003), astro-ph/0302483

[2] Biermann, L.F., Z. f. Naturf. 5a, 65 - 71 (1950)

[3] Biermann, L.F., Zeitschr. für Astrophys. 29, 274 (1951).

[4] Biermann, L.F., Schlüter, A., Phys. Rev. 82, 863 - 868 (1951)

[5] Biermann, P.L., Astron. \& Astroph. 271, 649 (1993), astro-ph/9301008.

[6] Biermann, P.L., at 23rd International Conference on Cosmic Rays, in Proc. Invited, Rapporteur and Highlight papers; Eds. D. A. Leahy et al., World Scientific, Singapore, p. 45 (1994).

[7] Biermann, P.L. \& Galea, C.F., invited review at the 9th course of the Chalonge School on Astrofundamental Physics: "The Early Universe and The Cosmic Microwave Background: Theory and Observations"; Ed. Norma Sanchez, in press (2003), astro-ph/0302168

[8] Biermann, P.L., Moiseenko, S., Ter-Antonyan, S., \& Vasile, A., invited review at the 9th course of the Chalonge School on Astrofundamental Physics: "The Early Universe and The Cosmic Microwave Background: Theory and Observations"; Ed. Norma Sanchez, in press (2003), astro-ph/0302201

[9] Bisnovatyi-Kogan, G.S., Astron. Zh. (Sov. Astron.), 47, 813 (1970)

[10] Bisnovatyi-Kogan, G.S., Moisennko, S.G., Sov. Astron., 36, 285 (1992)

[11] Coburn, W., Boggs, S.E., Nature 423, 415 - 417 (203)

[20] Cox, D. P., Astrophys. J. 178, 159 - 168 (1972)

[13] Hess, V.F., Phys. Z. 13, 1084 (1912).

[14] Jokipii, J. R., Astrophys. J. 313, 842 - 846 (1987).

[15] Hjorth, J. et al., Nature 423, 847 - 850 (2003)

[16] Kohlhörster, W., Phys. Z. 14, 1153 (1913).

[17] Kardashev, N. S., Astron. Zh., 41, 807 (1964)

[18] Lagage, P. O., \& Cesarsky, C. J., Astron. \& Astroph. 125, 249 - 257 (1983).

[19] Paczynski, B., in Proc. Gamma-Ray Burst: 4th Huntsville Symposium, Huntsville, AL Sept. 1997. Eds. by Ch. A. Meegan et al.. Woodbury, New York : AIP conference proceedings ; 428, p. 783 (1998)

[20] Parker, E. N., Astrophys. J. 128, 664 (1958)

[21] Parker, E. N., Astrophys. J. 157, 1129 (1969)

[22] Price, P.A. et al., Nature 423, 844 - 847 (2003)

[23] Seemann, H. \& Biermann, P.L., Astron. \& Astroph. 327, 273 (1997), astro-ph/9706117

[24] Snowden, S.L., et al., Astrophys. J. 485, 125 (1997).

[25] Steenbeck, M., Krause, F., Monatsb. Dt. Akad. Wiss. 7, 335 - 340, (1965), translated in NCAR-TN/IA-60 The turbulent dynamo, edited by P.H. Roberts, M. Stix, p. 21 (1971)

[26] Uemara, M., et al., Nature 423, 843 - 844 (2003)

[27] Völk, H. J. \& Biermann, P. L., Astrophys. J. Letters 333, L65 - L68 (1988) 
[28] Wiebel-Sooth, B., Biermann, P.L., Landolt-Brnstein: Numerical Data and Functional Relationships in Science and Technology - New SeriesGruppe/Group 6 Astronomy and Astrophysics, Volume 3, Ed. H.H. Voigt: Extension and Supplement to Volume 2, Interstellar Matter, Galaxy, Universe, vol. VI/3c, Springer Verlag, p. 37 - 90 (1999). 